Théologiques

Théologiques

\title{
Le rapport des humains aux animaux dans la perspective de l'éthique
}

\section{Mise en situation sociale}

\section{Denis Müller}

Volume 10, numéro 1, printemps 2002

Les animaux dans la conscience humaine

URI : https://id.erudit.org/iderudit/008157ar

DOI : https://doi.org/10.7202/008157ar

Aller au sommaire du numéro

\section{Éditeur(s)}

Faculté de théologie de l'Université de Montréal

\section{ISSN}

1188-7109 (imprimé)

1492-1413 (numérique)

Découvrir la revue

\section{Citer cet article}

Müller, D. (2002). Le rapport des humains aux animaux dans la perspective de l'éthique : mise en situation sociale. Théologiques, 10(1), 89-108.

https://doi.org/10.7202/008157ar

\section{Résumé de l'article}

Le statut éthique et juridique de l'animal fait l'objet de discussions intenses aujourd'hui. Sa résolution suppose des choix difficiles entre des modèles argumentatifs en apparence incompatibles. L'auteur compare les deux principales formes de biocentrisme, le biocentrisme égalitaire et le biocentrisme hiérarchique, à l'anthropocentrisme, qui peut lui-même être radical ou modéré. Il en découle que le modèle anthropocentrique présente des avantages décisifs, à condition de subir une révision fondamentale, dans le sens d'une anthropo-relationnalité qui distingue la domination violente de l'homme sur l'animal du respect pleinement humain — et donc aussi humanisant - envers les animaux, dont il importe de prendre en compte la dignité et la valeur. La voie du pathocentrisme trouve alors une certaine justification, mais sans occulter la perspective humaine seule à même de fonder une éthique et de la rendre socialement plausible. 


\title{
Le rapport des humains aux animaux dans la perspective de l'éthique Mise en situation sociale
}

\author{
Denis MülleR \\ Université de Lausanne
}

La question du statut éthique et juridique de l'animal est en plein renouveau ${ }^{1}$. Notre propos, dans cette contribution, est d'éclairer la discussion éthique en cours, en situant ses principaux enjeux anthropologiques. Cela nous conduira à dépasser l'opposition trop dure entre l'anthropocentrisme et le biocentrisme, en nous orientant à l'aide du concept d'anthropo-relationnalité. Nous tenterons ainsi de préciser les conséquences anthropologiques impliquées dans la relation éthique de l'homme avec les animaux.

\section{Le statut de l'animal}

Deux modèles s'affrontent prioritairement dans la discussion au sujet du statut éthique de l'animal. D’un côté, l'accent est placé sur les droits des animaux, concept qui prend la plupart du temps, dans cette optique, une signification métaphorique ou dérivée. D’un autre côté, on insiste davantage sur le respect dû aux animaux, que ce respect soit fondé sur un respect général de la vie ou de la nature,

1. Voir les études réunies dans D. Müller et H. Poltier, dir, La dignité de l'animal. Quel statut pour les animaux à l'heure des technosciences? (Le champ éthique 36), Genève, Labor et Fides, 2000. Le présent article, prolongeant ces études, est le fruit partiel d'un mandat de la Confédération helvétique, effectué avec le précieux concours de ma collègue Andrea Arz de Falco (Université de Fribourg). Cf. notre ouvrage commun Les animaux inférieurs et les plantes ontils droit à notre respect? Réflexions éthiques sur la dignité de la créature, Genève, Médecine et hygiène, 2002. 
ou qu'on le fonde sur une dignité spécifique ou sur une valeur intrinsèque de l'animal.

Le langage des droits fait problème. Un philosophe contemporain, Jean-Yves Goffi, qui fait pourtant un usage majeur de cette notion dans son plaidoyer éthique en faveur des animaux, plaidoyer mené au nom d'un pathocentrisme gradualiste ${ }^{2}$, doit lui-même reconnaître que seule une formulation faible de l'idée de tels droits permet de l'appliquer valablement aux animaux et aux humains ${ }^{3}$. Par ailleurs, cette formulation faible débouche sur la notion de valeur inhérente de l'animal, introduite par Tom Regan aux États-Unis et reprise notamment par Verhoog ${ }^{4}$ et Goffi en Europe.

Les limites du discours faisant état de droits des animaux sont assez clairement établies, en tout cas en ce qui concerne leur formulation forte. Ne doit-on pas admettre, en effet, que seuls sont susceptibles de détenir des droits les êtres capables d'exercer également des devoirs envers autrui ? Malgré les efforts louables de la communauté scientifique internationale, il n'a jamais été possible d'attribuer une telle capacité à un animal, même pas aux chimpanzés ou aux bonobos, pourtant supposés disposer de quelque vocabulaire élémentaire à défaut de pouvoir élaborer une grammaire. Il ne suffirait nullement, en effet, que l'on puisse démontrer l'existence, chez tel animal supérieur, d'une véritable capacité linguistique et d'un seuil minimal de conscience cognitive et de conscience de soi; encore faudrait-il que l'on puisse faire état de la présence d'une conscience morale chez le même animal. La difficulté n'est pas d'abord épistémologique; elle consiste plutôt dans la question de savoir s'il est possible d'attribuer à l'animal des capacités réflexives et morales

2. Le pathocentrisme gradualiste suppose qu'il y a certains degrés dans la souffrance et qu'il convient d'en tenir compte notamment dans une appréciation éthique.

3. J.Y. Goffi, «Des droits à la valeur: la question des êtres de nature", dans Müller et Poltier, La dignité de l'animal, p. 249-258, 249. Voir les analyses très approfondies du même auteur dans son livre Le philosophe et ses animaux. Du statut éthique de l'animal, Nîmes, Éd. J. Chambon, 1994.

4. H. Verhoog, "La bioéthique et la "nature" des animaux ", dans Müller et Poltier, La dignité de l'animal, p. 237-248. 
compréhensibles et si, de plus, une telle attribution ne résulterait pas d'une projection anthropomorphique.

Les apories inhérentes à l'idée de droits des animaux sont donc très révélatrices de la nécessité de clarifier le statut du point de vue moral comme tel; ce n'est pas seulement le concept de conscience morale qui pose difficulté à cet égard, mais aussi l'usage de catégories éthiques centrales comme celles de réciprocité ou d'intersubjectivité. Il apparaît de plus en plus évident qu'à moins d'adopter une théorie radicalement naturaliste ou sociobiologique de l'éthique, la référence à des droits de l'animal demeure une évocation de type métaphorique, certes utile pour signaler la revendication d'un respect dû aux animaux, mais impossible à intégrer harmonieusement dans une éthique réflexive et critique.

C'est bien pourquoi la problématique du respect de la vie est à notre avis plus prometteuse et plus réaliste, à condition de ne pas succomber à une conception absolue d'un tel respect et de ne pas sacrifier à une forme de vitalisme ou de naturalisme. L'enjeu central, dès lors, est celui des relations entre le vivant et l'humain. Comment l'être humain peut-il, dans le cadre du respect général qu'il se reconnaît devoir à la vie et à la nature, en venir à respecter la dignité ou la valeur de l'animal? Qu'est-ce que cela change pour son comportement quotidien et pour la structuration de l'espace social autour de lui ? Comment peut-il être davantage conscient au plan moral et mieux équipé au plan socio-politique pour affronter les défis que représentent aujourd'hui les biotechnologies et leur environnement commercial orienté sur la concurrence et le profit?

De tels enjeux pratiques et politiques impliquent la solution de plusieurs difficultés théoriques de grande envergure. C'est vers certaines d'entre elles que nous allons nous tourner avant tout dans les remarques qui suivent.

\section{Biocentrisme et anthropocentrisme}

Cette première problématique a été très souvent associée à l'héritage judéo-chrétien, généralement accusé d'être à la source et en faveur de l'anthropocentrisme le plus décidé et le plus pernicieux. 
La cause paraît entendue à bien des auteurs traitant la question du statut éthique de l'animal: il faudrait opter sans l'ombre d'une hésitation pour une posture biocentrique et même pathocentrique ${ }^{5}$ et en finir une fois pour toutes avec le préjugé anthropocentrique.

On retrouve cette opposition derrière l'alternative des droits ou de la dignité de l'animal. Les animaux détiennent-ils des droits propres ou une valeur inhérente, totalement indépendante de la présence et du regard même de l'homme, ou faut-il admettre que même leurs droits ou leur valeur sont à dériver d'une dignité que l'homme accepte de leur attribuer ou de leur reconnaître? Plus largement, on s'interroge ici sur l'idée même que la nature, comme telle, aurait ou non des droits.

\subsection{La discussion philosophique}

Un philosophe allemand contemporain, Klauspeter Rippe ${ }^{6}$, a récemment discuté les avantages respectifs du biocentrisme égalitaire, défendu par Paul Taylor ${ }^{7}$ et James Sterba, et du biocentrisme hiérarchique, auquel Rippe accorde sa préférence.

À lire Rippe, et malgré ses efforts louables pour rendre justice à Taylor, force est de constater les impasses d'un tel biocentrisme égalitaire. Rippe résume ainsi la thèse centrale de ce dernier: «Les biocentristes égalitaires prétendent que les êtres vivants ne sont pas seulement égaux en vertu du fait qu'ils ont une valeur inhérente, mais aussi parce que leur valeur inhérente est de même valeur ${ }^{8}$.» Il

5. La posture biocentrique donne la priorité au vivant sous toutes ses formes, tandis que la posture pathocentrique privilégie le critère de la souffrance expérimentée par les êtres vivants (animaux compris). Il y a donc à la fois une proximité et une différence entre les deux approches. Par ailleurs, elles s'efforcent toutes les deux de surmonter les impasses de l'anthropocentrisme.

6. K. Rippe, «Les êtres vivants sont-ils tous de valeur égale. À propos de la discussion entre le biocentrisme égalitaire et le biocentrisme hiérarchique ", dans Müller et Poltier, La dignité de l'animal, p. 259-280.

7. Voir son ouvrage majeur Respect for Nature: A Theory of Environmental Ethics, Princeton, Princeton University Press, 1989². Sur P. Taylor, voir aussi Goffi, Le philosophe et ses animaux, p. 235ss.

8. Rippe, «Les êtres vivants», p. 260. 
ne découle cependant pas de cette affirmation que tous les êtres vivants doivent être traités d'une manière égale. Selon les situations, nos devoirs peuvent varier, sans léser le principe de justice inhérent à l'égalité. "Le biocentrisme égalitaire dit seulement qu'il y a des devoirs déterminés que nous avons envers tous les êtres vivants ${ }^{9}$ » et que, par ailleurs, tous les êtres vivants ont droit à de justes traitements, dans le sens d'un égal respect.

Le biocentrisme hiérarchique, selon Rippe, conteste ces deux assertions. Les deux camps sont contre le recours à l'appartenance à l'espèce comme unique légitimation morale, mais les tenants du biocentrisme hiérarchique sont d'avis que «le privilège accordé à certaines espèces ou à certains règnes repose sur des distinctions moralement pertinentes ${ }^{10} »$. Le biocentrisme hiérarchique n'est pas dualiste: il préfère parler d'une échelle de la nature, ce qui permet de faire varier la positivité normative des différentes espèces ou entités considérées.

Le biocentrisme hiérarchique présente des avantages cognitifs et éthiques. Il correspond mieux à nos intuitions, aussi bien à nos intuitions pratiques (dans nos relations réelles avec tel ou tel animal) qu'à nos intuitions morales (nous mangeons plus volontiers de la vache que du chien, en Occident). L'argument décisif touche la priorité accordée à l'homme sur l'animal. On le voit bien dans le devoir d'assistance, qui varie évidemment selon que c'est un être humain ou un animal qui est menacé de mort ou que la situation (un incendie, par exemple) nous met en demeure de choisir.

Les biocentristes égalitaires émettent à cet égard deux objections. D'une part, disent-ils, la thèse de la priorité tend à justifier l'usage immoral et arbitraire des animaux. D'autre part, l'idée d'échelle de la nature est elle-même contingente et arbitraire.

Rippe se rallie néanmoins, au terme d'une argumentation très détaillée, à l'idée selon laquelle le biocentrisme hiérarchique est possible et nécessaire. Il est possible, parce que nos intuitions ne

9. Rippe, "Les êtres vivants ", p. 263.

10. Rippe, "Les êtres vivants", p. 263. 
parlent pas dans le sens d'un égalitarisme strict entre tous les êtres vivants, ni du point de vue factuel, ni du point de vue éthique ou normatif. Le biocentrisme hiérarchique dépasse de ce fait la confession de foi purement rhétorique et donc inapplicable du biocentrisme égalitaire. Le biocentrisme hiérarchique est dès lors nécessaire, parce qu'il exige "une considération morale de tous les êtres vivants ${ }^{11}$ ", surmontant le statu quo par sa manière d'abandonner clairement l'anthropocentrisme, lequel a tendance, au contraire, à exclure certains êtres vivants de toute considération morale.

Le plaidoyer nuancé de Rippe en faveur du biocentrisme hiérarchique a le mérite de montrer sans ambiguïté les impasses du biocentrisme égalitaire. Il ne me semble cependant pas que les arguments de Rippe en faveur du biocentrisme hiérarchique aient produit de sérieuses raisons de renoncer à toute forme d'anthropocentrisme. Nous ne sommes nullement obligés, d'abord, de lier l'anthropocentrisme à l'exclusion systématique de toute considération morale des animaux. Ensuite, l'idée de hiérarchie présuppose nécessairement la supériorité à la fois cognitive et éthique d'une perspective de type anthropocentrique. Le seul argument susceptible d'ébranler la thèse anthropocentrique consisterait dans le fait de soutenir que le biocentrisme hiérarchique, contrairement à l'anthropocentrisme, exigerait un égal respect éthique de tous les êtres vivants et que, par conséquent, toute forme d'anthropocentrisme serait discriminatoire. Or cet avantage supposé du biocentrisme éclairé ne tient la route qu'à la condition de lui opposer un anthropocentrisme radical et extrême, dépourvu lui-même de l'autocritique immanente dont il nous paraît pourtant susceptible. C'est pourquoi nous en appellerons de notre côté à un anthropocentrisme modéré ou mitigé, dûment éclairé et corrigé par la critique. Cet anthropocentrisme modéré aura à se penser comme une attitude de respect centrée sur la relation responsable de l'homme avec les autres êtres vivants. À la limite, nous abandonnerons le langage de l'anthropo-centrisme, trop connoté négativement, pour lui substituer celui de l'anthropo-relationnalité,

11. Rippe, «Les êtres vivants », p. 277. 
plus convivial et plus responsable. Mais, comme nous le verrons, cette relationnalité ne supprime nullement la primauté de l'être humain; elle est fondamentalement a-symétrique, ce qui la rapproche forcément de l'intention la plus positive de l'anthropocentrisme. Elle suppose la reconnaissance de la différence anthropologique et du caractère spécifiquement humain du point de vue moral lui-même.

\subsection{Le procès $d u$ christianisme}

Il nous faut nous arrêter à la fonction réelle ou supposée du christianisme dans ce débat. Nous ne le faisons pas seulement en tant que théologien, mais parce que nous estimons que cette question est déjà incontournable sous le simple point de vue de l'histoire et de la culture.

Dans sa version vulgarisée de la libération animale, comme dans sa conception utilitariste plus générale de l'éthique pratique, Peter Singer a instruit le procès du christianisme et de ses sources juives comme principal responsable de la maltraitance des animaux ${ }^{12}$. Cette idée est reprise à bien des égards par Élisabeth de Fontenay dans ses travaux ${ }^{13}$, mais elle le fait en se situant, elle, aux antipodes de tout utilitarisme, dont le caractère sacrificiel est précisément en cause $^{14}$.

Lynn White Jr, historien de la pensée médiévale, avait présenté en 1967 les pièces du dossier ${ }^{15}$. Quelles étaient alors ses principales observations et ses conclusions?

12. P. Singer, La libération animale / trad. par L. Rousselle, Paris, Grasset, 1993 (anglais 1975).

13. É. De Fontenay, Le silence des bêtes. La philosophie à l'épreuve de l'animalité, Paris, Fayard, 1998. Voir par ailleurs, sous forme condensée, ses deux contributions dans l'ouvrage cité de Müller et Poltier.

14. Sur les liens entre utilitarisme et sacrifice, $c f$. D. MülleR, "Utilitarisme et christianisme. Une triple mise en perspective", dans Les passions de l'agir juste. Fondements, figures, épreuves, Fribourg / Paris, Éditions Universitaires / Le Cerf, 2000, p. 69-80.

15. L. White, "Les racines historiques de notre crise écologique ", dans Goffi, Le philosophe et ses animaux, p. 289-309. À l'origine, il s'agit d'un article paru dans Science. Sur le sujet, cf. M. DAmien, L'animal, l'homme et Dieu, Paris, Le 
White montrait que la victoire du christianisme sur le paganisme a été la plus grande révolution psychique de notre histoire culturelle. Nos façons quotidiennes d'agir envers l'environnement ont leurs racines dans la "téléologie judéo-chrétienne ${ }^{16}$ ", et uniquement en elle, comme l'attesta le fait que le marxisme - cette hérésie judéochrétienne - n'eut aucune difficulté à la reprendre intégralement.

Selon White, les traits distincts du judéo-christianisme sont éloquents, si l'on pense à la vision grecque d'un Aristote: la Bible pense le monde en terme de commencement; l'homme y est à l'image de Dieu; il reçoit de Dieu le pouvoir de nommer les animaux et les plantes; l'homme est appelé à vivre dans la suivance (à la suite) du Christ, ce second Adam, qui transcende lui aussi la nature par sa résurrection. Bref: il n'y a pas de religion plus anthropocentrique que le christianisme, notamment dans sa forme occidentale. Deux traits corrélatifs en ressortent: un dualisme entre l'homme et la nature, et une volonté, de la part de Dieu, que l'homme exerce sa domination sur cette nature et l'exploite à ses propres fins ${ }^{17}$.

White était cependant obligé de reconnaître que l'exploitation de la nature résulte davantage du christianisme latin que du christianisme oriental et byzantin, plus intellectualiste et moins volontariste. Néanmoins, dans le procès intenté par White au christianisme, la part des choses ne nous paraît pas établie de manière différenciée et équitable: il est en effet assez simplificateur de déduire la naissance de la technologie et de la science modernes directement du christianisme, comme si d'autres facteurs n'avaient pas joué leur rôle.

Il n'est guère étonnant de voir White, à la fin de son article, en appeler à un autre christianisme, placé sous le patronage de SaintFrançois d'Assise. White était en effet convaincu, en bon Américain du Nord, que seule la religion peut corriger et amender ce que la religion a dénaturé.

Cerf, 1978; A. De Pury, Homme et animal Dieu les créa. Les animaux et l'Ancien Testament, Genève, Labor et Fides, 1993; A. Bondolfi, L'homme et l'animal. Dimensions éthiques de leur relation, Fribourg, Éditions Universitaires, 1995.

16. White, «Les racines historiques », p. 300.

17. White, «Les racines historiques », p. 301. 
Tous les auteurs qui font du christianisme, si je puis dire, le bouc émissaire de la crise écologique et du mépris envers les animaux, ne sont à vrai dire pas aussi généreux et aussi remplis d'espoir envers la religion que White ou que le sera à notre époque - non sans ambiguïtés - un théologien comme Eugen Drewermann ${ }^{18}$.

Dans l'approche critique de la philosophe Élisabeth de Fontenay, le christianisme apparaît centralement comme une puissance christique de symbolisation et de métaphorisation des animaux, au détriment de leur sort réel dans l'histoire et dans la société. Le fait que le christianisme ait aboli les sacrifices animaux a eu selon cette auteure l'effet paradoxal et pervers de conduire à une "appropriation sans foi ni loi de la nature et même de ceux qui, en elle, s'apparentent plus à l'humain qu'au végétal [...]. La souffrance animale n'a plus aucune signification puisqu'elle n'a pas été comptée dans le rachat et que les bêtes sont de moins en moins animées et donc animales, qu'elles ont perdu leur destin d'âmes ${ }^{19}$. " Soucieuse de traiter le christianisme avec équité, Élisabeth de Fontenay, tout en accablant saint Augustin et l'inanité de son argumentation contre l'idée d'une souffrance des animaux ${ }^{20}$, reconnaît que le christianisme a donné néanmoins des signes de «repentir» — de saint François d'Assise à Paul Claudel et Léon Bloy. Le «triomphe de l'agneau " n'a pas reproduit sans autres «le temps des sacrifices» et n'a pas réussi à faire complètement oublier la mauvaise conscience suscitée par la question somme toute assez rhétorique de l'apôtre Paul: «Dieu prend-il soin des bœufs? » (1Co 9,9) Le sujet «masculin, parlant et carnivore ", dénoncé à la suite de la critique de la métaphysique occidentale par Jacques Derrida, n'est-il pas une création symptomatique du christianisme, dont la fonction historiale est, finalement, de «noyer le poisson» au prix d'une métonymie subtile? Nous serions

18. E. Drewermann, De l'immortalité des animaux / trad. par B. Lauret, Paris, Le Cerf, 1992 (allemand 1990). Voir ma critique: "Une éthique de l'immédiateté ", dans Clés pour comprendre Drewermann. L'actualité religieuse dans le monde, Hors-Série 1, 1993, p. 46-49.

19. Fontenay, Le silence des bêtes, p. 250.

20. Fontenay, Le silence des bêtes, p. 268. 
parvenus ainsi à une véritable «intériorisation du sacrifice », de type clairement utilitariste, soumis à la logique du marché, de la «grande bouffe" au "grand massacre ». Élisabeth de Fontenay en appelle dès lors à "réinscrire l'animal dans une chaîne symbolique qui ne fasse plus bon marché de lui ${ }^{21}$ ». Toute porte n'est donc pas fermée pour une éventuelle reprise théologique de la question. Avouons cependant que, dans l'ensemble, la charge d'É. de Fontenay est impitoyable.

Il ne fait pas doute que le procès du christianisme instruit de cette manière, s'il peut paraître excessif et sélectif, lance un défi aussi bien aux Églises et aux théologiens qu'à l'ensemble des sociétés postchrétiennes dans lesquelles nous vivons aujourd'hui.

Un des enjeux principaux exige selon nous de surmonter les impasses de l'anthropocentrisme radical, mais sans rien céder sur la responsabilité singulière des êtres humains. En ce sens, nous en appelons de notre côté à une nouvelle vision anthropologique, centrée sur la relation respectueuse et responsable de l'homme avec l'animal. À notre avis, une telle vision est tout à fait compatible avec une relecture critique et une reconstruction moderne de l'éthique théologique d'inspiration juive et chrétienne, comme il conviendrait de le démontrer bien évidemment plus en détail.

Nous ne sommes pas en mesure de proposer ici les mesures concrètes découlant d'une telle vision. Nous nous contentons de proposer un cadre théorique général. Nous pensons en effet très important que s'opère déjà un changement de mentalité, auquel les Églises et les chrétiens ont la possibilité et le devoir de contribuer. Précisons de plus, pour éviter de susciter de faux espoirs, que le modèle envisagé ici, invitant à une relation plus respectueuse et plus responsable envers les animaux, va davantage dans le sens d'une pesée des intérêts entre les biens humains et les biens animaux que dans celui d'un moratoire ou d'une interdiction totale de certaines pratiques sociales; en clair, nous ne suivons pas un Peter Singer, un Eugen Drewermann ou une Élisabeth de Fontenay lorsque, sur des bases philosophiques ou théologiques diverses, ils en viennent (de

21. Fontenay, Le silence des bêtes, p. 716. 
manière la plupart du temps explicite) à exiger un arrêt de la consommation de viande ou une cessation absolue des expériences sur les animaux. De telles propositions, extrêmes et radicales, nous semblent à la fois éthiquement illégitimes et socialement impraticables: même si nous les estimions souhaitables, elles n'ont que très peu de chances de se réaliser démocratiquement et historiquement. Il n'appartient pas à l'éthique théologique chrétienne de proposer des utopies totalement inaccessibles (ce serait confondre théologiquement l'avènement définitif du Royaume avec la réalité historique). Par contre, nous devons relever ces défis avec un authentique sérieux et accepter de soumettre nos conceptions du monde à une critique théorique et sociale très approfondie. La perspective eschatologique du Royaume de Dieu pourra nous servir ici de détonateur critique très puissant. Les modestes réflexions qui suivent entendent baliser une approche renouvelée de la question, à la fois sur une base théologique et dans une visée de critique culturelle et sociale.

\section{Les limites du modèle taxinomique et le principe anthropologico-relationnel comme cadre de référence de la classification animale}

Les efforts contemporains pour échapper aux effets négatifs de l'anthropocentrisme favorisent le retour de conceptions plus holistiques et plus amicales envers l'environnement. À quoi ressemble par exemple l'échelle moderne de la nature (l'antique idée de scala naturae, reprise dans le modèle du biocentrisme hiérarchique discuté plus haut) qui sert de guide implicite dans les tentatives législatives de réformes du statut des animaux? Elle entend gérer et concilier symboliquement et pratiquement deux choses d'apparence antagoniste: une analogie permettant de différencier sans céder à la rupture (le bonobo, par sa grande ressemblance avec l'être humain, est en même temps l'animal qui érige l'être humain en être humain différent, différent du bonobo surtout), mais aussi un mode d'intervention de l'humain, mode auquel se voit reconnaître une certaine légitimité morale en même temps qu'une nécessaire limite (l'animal domestique dit la trace et porte la marque du dresseur, par exemple; mais 
sa domestication dit aussi son humanisation et l'bumanisation de l'homme qu'elle appelle en retour).

Nous sommes ainsi conduits à reconnaître une certaine priorité méthodologique et épistémologique à nos intuitions morales, fondées sur des principes comme la liberté et la justice. Ces intuitions, mises en avant par divers auteurs, ne relèvent cependant pas pour nous d'un biocentrisme hiérarchique ou d'une éthique de l'écologie profonde enracinée dans un concept englobant de nature, mais d'une acceptation critique de l'option anthropocentrique. Notre intention est ici de montrer comment la perspective centrée sur la «différence anthropologique» (expression de Frank Tinland ${ }^{22}$ ) ou sur la notion d'anthropo-relationnalité (employée par Dietmar van der Pfordten ${ }^{23}$ ) permet de dépasser les impasses d'un simple anthropocentrisme sans pour autant ratifier le pathocentrisme ou le biocentrisme.

Certes, la notion d'intuition est encore trop pauvre et trop formelle pour prendre en compte la richesse de nos expériences historiques et culturelles. Notre rapport éthique aux animaux (sans parler du monde des plantes) n'est pas structuré uniquement par l'intuition d'une valeur ou d'une dignité qui leur seraient inhérentes, en fonction de la manière dont la science empirique rendrait compte de leur singularité. Il découle de manière infiniment plus profonde de notre expérience historique et culturelle avec la diversité du monde animal, aussi bien dans la singularité de leur existence individuelle que dans l'épaisseur de leur présence environnementale.

Nous buttons ici sur une première difficulté méthodologique et épistémologique, qui n'est pas sans incidences, il faut le souligner, sur notre réflexion éthique proprement dite. Où passe la frontière certains parlent d'une zone grise - entre les animaux prétendus supérieurs et les animaux prétendus inférieurs? Et pour autant qu'une telle barrière existe et puisse être formulée de manière perti-

22. F. Tinland, La différence anthropologique. Essai sur les rapports de la Nature et de l'Artifice, Paris, Aubier-Montaigne, 1977.

23. D. Van der Pfordten, Ökologische Ethik, Hamburg, Rowohlt, 1996, p. 63. L'auteur plaide pour des «anthroporelationale Begründungen normativer Ethik». 
nente, quelle est sa portée normative et éthique? De quelle nature serait une telle hiérarchie? La supériorité et l'infériorité en question représentent-elles des notions descriptives ou des notions normatives? Comment penser l'originalité irréductible du point de vue humain sans succomber à une conception dominatrice et prédatrice, encore trop sous-jacente dans l'idée que nous nous faisons de la hiérarchie?

L'enjeu ultime n'est pas "Qui domine?» mais «Comment la responsabilité éthique envers les différents types d'êtres vivants se situe-t-elle dans la perspective de l'être humain »?

Un tel renversement de perspective relativise l'importance de la classification zoologique ou, pour reprendre les termes d'une ethnologue contemporaine ${ }^{24}$, il réoriente l'idée de classification objective en direction d'un ordre des êtres vivants reconnaissant à chacun une place significative. La notion de "classe» émet une prétention objectivante constamment remise en question, tandis que l'idée de «place» est plus relationnelle et s'avère en même temps compatible avec une certaine primauté de la perspective humaine.

Cette optique de l'anthropo-relationnalité ou de la différence humaine permet d'échapper à la déduction pure et simple des catégories éthiques à partir des niveaux ou des degrés de la nature. «Toute la question est [...] de savoir si la singularité anthropologique se laisse mieux penser à partir d'une différenciation interne de la nature (en "niveaux") ou à partir d'une différence entre le naturel et autre chose, différence qui n'exclurait pas la dépendance de cette "autre chose" par rapport à ce qui demeurerait en deçà de la coupure ${ }^{25}$.»

Dans le discours zoologique dominant, il nous paraît possible de constater deux choses. D'une part, la possibilité même d'une classification horizontale, portant sur les animaux actuellement observables et répertoriés, est fortement mise en question et reconnue

24. M. Albert-Llorca, L'ordre des choses. Les récits d'origine des animaux et des plantes en Europe, Paris, CTHS, 1991, p. 270.

25. Tinland, La différence anthropologique, p. 440. 
comme problématique. D'autre part, tout effort taxinomique semble subordonné au regard vertical de l'évolution, comme le montre notamment le recours à la paléontologie.

Cependant, il convient de mettre ici un bémol sur la prédominance des modèles scientifiques dans l'argumentation éthique. Donnons un exemple très simple, qui parle directement à notre sens commun: l'évidence scientifique selon laquelle l'homme et le singe descendent l'un et l'autre des reptiles ne permet pas de déduire que l'homme, le singe et le serpent appellent de notre part un même respect ou possèdent la même dignité. De même, la plus grande proximité génétique du dauphin et de la vache, par rapport à celle que l'on peut semble-t-il constater entre la vache et le cheval, n'est guère porteuse de conséquences éthiques quant au respect dû à ces différents mammifères. Pas davantage que la génétique, l'évolution ne saurait commander l'éthique; une éthique évolutionniste ne signifie pas nécessairement la domination du point de vue de l'évolution sur nos intuitions éthiques ou sur le point de vue éthique en tant que tel.

Selon notre perspective normative, le point de vue éthique est centré, telle une ellipse à double foyer, sur l'affirmation de la liberté humaine et sur l'exigence de justice, justice qui s'applique de manière différenciée à l'ensemble des êtres vivants. Ce n'est donc pas l'origine de l'homme comme être vivant singulier qui détermine le sens de l'éthique, mais l'éthique qui oriente le regard de l'homme sur le monde. Le point de vue moral (moral point of view) cher aux philosophes anglo-saxons se voit ainsi reconnu pour ce qu'il est: non pas une règle formelle relevant de la méta-éthique, mais la résultante d'une prémisse anthropo-relationnelle pure, qu'il est impossible et inutile de vouloir rapporter à un prétendu fondement naturel ou biologique.

Dans le même ordre de pensée, le respect envers les animaux et les plantes ne peut pas se déduire purement et simplement d'une table de classification de type zoologique ou botanique, comme si l'éthique avait à épouser de manière automatique le mouvement de différenciation et d'évolution des êtres vivants. Sans compter le 
caractère toujours problématique et fondamentalement mobile de tels efforts taxinomiques, l'appartenance à une même espèce ou à une même classe ne suffit pas à elle seule à fonder une égalité de respect (c'est la part de vérité de la critique du spécisme). Deux mammifères, par exemple, ne sont pas nécessairement appelés à recevoir un égal respect, du seul fait qu'ils sont des mammifères. $\mathrm{Ou}$, pour prendre à dessein un exemple plus culturel que zoologique, un animal domestique et un animal sauvage ne doivent pas nécessairement être traités de manière égale, en ce qui concerne leur mise en liberté ou leur tenue en captivité. On tiendra compte de leur dangerosité et du contexte propre à la réduire au mieux. Si on veut traiter un tigre de manière égale à un chien, on relâchera le tigre dans la jungle, ou alors on acceptera qu'il demeure en cage. Le fait de le mettre en cage n'est pas en soi et pour soi une atteinte à sa dignité. On voit combien la pesée éthique des intérêts dépend toujours des circonstances.

\section{Dignité et valeur. La discussion éthique}

Mais nous aimerions relancer l'interrogation d'une manière encore plus pointue. Comme nous en avons récemment fait l'expérience dans la discussion nationale en Suisse, où la Constitution fédérale postule l'idée d'une dignité de la créature ${ }^{26}$ s'appliquant aux animaux et aux plantes, il paraît nécessaire de conserver à cette catégorie de dignité de la créature un maximum - certains diront seulement: un minimum — de pertinence et d'extension. Cela ne s'est pas produit sans certaines distorsions sémantiques, voire sans certaines jongleries verbales, qui ne nous paraissent pas propices à une bonne compréhension des enjeux éthiques en présence. Nous nous risquons donc ici, afin de faire progresser le débat, à clarifier les termes de la discussion, en procédant à une distinction plus nette entre le langage de la dignité et celui de la valeur.

Le passage du langage de la dignité au langage de la valeur justifie-t-il de conserver le concept de dignité de la créature comme

26. Voir le dossier réuni par Müller et Poltier, La dignité de l'animal. 
concept supérieur s'appliquant aux animaux, voire aux plantes? En procédant de la sorte, ne risque-t-on pas de le vider de sa substance et de sa signification première et de n'en faire plus qu'une black box sans pertinence et sans plausibilité ?

Il est bien évident que notre attachement au concept de dignité de la créature obéit pour une part importante à des impératifs historiques et pragmatiques (cela nous conduit à parler de la fonction métaphorique du concept de dignité de la créature). Cependant, il ne découle pas de ces impératifs que nous devions nous en tenir à une conception dogmatique, étroite et rigide d'un tel concept. D'une part, l'histoire même du concept et de son interprétation, tant juridique que politique et intellectuelle, atteste de sa flexibilité. D’autre part, l'analyse interne du concept montre de manière suffisamment convaincante qu'il est compatible avec une "déclinaison graduelle » de sa pertinence et de ses applications. Cette déclinaison ne doit pas craindre d'indiquer clairement les moments et les lieux où il convient de renoncer au concept de dignité de la créature. Chacun comprendra d'ailleurs très bien qu'un concept devient un fourre-tout ou une métaphore usée (Nietzsche), lorsqu'il est utilisé sans discernement et sur un mode élastique et vague. D'où notre proposition: seule la restriction du concept de dignité de la créature aux animaux supérieurs (les mammifères avant tout) est susceptible de renforcer la crédibilité et la plausibilité d'un concept aussi controversé.

\section{Les avantages pratiques et sociaux de la solution anthropo-relationnelle}

Reconnaître que la dignité de la créature animale lui confère une valeur spécifique, non identique à celle de l'être humain, mais admettre aussi que tous les animaux n'ont pas la même portée affective, esthétique, culturelle et environnementale aux yeux de l'être humain, c'est consacrer le primat relatif de l'être humain, de son histoire et de sa culture, mais en même temps lui assigner un impératif éthique de respect et de responsabilité envers l'animal et sur la nature. Le conditionnement anthropo-relationnel de l'être humain comme être socio-éthique le rend d'autant plus responsable envers ce 
qui fait à la fois le cadre et la visée de son activité humaine. Il a une dette envers ce dont il dépend, au nom même de la liberté et de la justice dont il se réclame et qui lui permette de transcender toute dépendance absolue.

La solution anthropo-relationnelle prend aussi ses distances avec la perspective pathocentrique radicale que très souvent, à la suite de Jeremy Bentham ${ }^{27}$, on voudrait imposer comme cadre de référence, en vertu d'une interprétation limitative et extrémiste du biocentrisme. Le pathocentrisme a ceci de particulier: tout en nivelant tous les êtres vivants au seul critère de la souffrance, il en revient de fait à projeter sur l'animal une vision anthropologique de la souffrance. Le pathocentrisme paraît ainsi difficilement échapper aux pièges de l'anthropomorphisme, à moins de limiter la souffrance à une notion purement objective et quantitative de douleur physique, ce qui semble fort réducteur.

Mais la perspective de l'anthropo-relationnalité n'est-elle pas elle-même entachée d'un certain pathocentrisme? N'est-elle pas obligée de recourir elle aussi au critère de la souffrance pour distinguer les traitements que l'être humain, dans sa relation à l'animal, estimera légitimes ou illégitimes de lui appliquer? Ne retombe-t-on pas dans les difficultés signalées à propos du biocentrisme égalitaire, où l'on est bien obligé d'introduire des différences de degré dans la douleur et dans la souffrance entre les animaux et les hommes, et donc de nous orienter plutôt dans le sens du biocentrisme hiérarchique?

Une solution de ce dilemme ne nous paraît possible qu'à la condition de lever le piège de l'anthropomorphisme et reconnaître honnêtement la primauté des intérêts humains sur les intérêts animaux. La souffrance supposée des animaux ne sera jamais, par-delà toute projection subjective et par-delà tout calcul de type quantitatif, qu'une estimation effectuée par l'homme. C'est à ce dernier qu'il appartient, dans sa relation effective avec l'animal, de juger quel

27. Le texte est cité dans le recueil de Bondolfi, L’homme et l'animal, p. 73: «La question n'est pas: peuvent-ils raisonner? Ni: peuvent-ils parler? Mais bien: peuvent-ils souffrir?» 
niveau de douleur et de souffrance il est prêt à accepter individuellement et socialement de faire subir à l'animal et quels moyens pratiques - techniques, mais aussi administratifs, juridiques et politiques — il est décidé à se donner pour réduire et limiter cette douleur et cette souffrance en la maintenant à son seuil le plus faible et plus respectueux de l'animal.

La perspective anthropo-relationnelle renvoie ainsi à la faculté de juger et à la responsabilité de l'être humain et de la société. Ses avantages sont puissants : elle renonce à l'attitude hypocrite consistant à se réfugier derrière des critères ou des mesures quantatitatifs ou objectifs pour décider du seuil acceptable de souffrance animale. Elle reconnaît de ce fait la douleur et la souffrance comme des critères partiels et subjectifs, devant être mis en balance avec d'autres critères, relevant d'autres intérêts et d'autres valeurs, en particulier les intérêts et les valeurs structurant le monde éthique socio-humain.

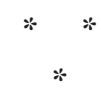

La perspective anthropo-relationnelle en appelle ainsi à un respect pleinement humain de l'animal, obligeant la société à se concerter et à se mettre d'accord sur les seuils de tolérance, de jugement et de responsabilité qu'elle est prête à se fixer dans sa relation différenciée avec les animaux comme individus singuliers et avec le monde animal plus généralement. Cette perspective est la plus proche du point de vue éthique tel que nous le comprenons. Elle n'a rien d'une prétendue réponse scientifique et objective, relevant de l'illusion scientiste et positiviste encore solidement implantée dans nos mentalités, mais elle rend compte du processus mental et de l'émergence sociale du sentiment de respect et du sens de la responsabilité qui font la grandeur et la dignité de l'homme et attestent de sa sociabilité. De ce point de vue, on dira que l'éducation anthropo-relationnelle au respect et à la responsabilité de l'homme envers l'animal, comme l'avait déjà signalé en son temps Emmanuel Kant, est un test déterminant de la dignité même de l'homme et de la qualité éthique de 
toute société humaine. La dignité de la créature animale oblige l'être humain à se structurer lui-même comme un être éthique, ouvert à une relation de non-maîtrise absolue et d'humilité envers les autres êtres vivants qui constituent son environnement. Elle a un impact social et politique évident. Elle nous paraît rejoindre, à son meilleur, la perspective centrale d'une éthique écologique d'inspiration juive et chrétienne, fidèle à l'héritage biblique, mais consciente aussi des déviances et des errements que ses lectures dominantes traditionnelles ont pu et pourraient encore générer.

\section{RÉSUMÉ}

Le statut éthique et juridique de l'animal fait l'objet de discussions intenses aujourd'hui. Sa résolution suppose des choix difficiles entre des modèles argumentatifs en apparence incompatibles. L'auteur compare les deux principales formes de biocentrisme, le biocentrisme égalitaire et le biocentrisme hiérarchique, à l'anthropocentrisme, qui peut lui-même être radical ou modéré. Il en découle que le modèle anthropocentrique présente des avantages décisifs, à condition de subir une révision fondamentale, dans le sens d'une anthropo-relationnalité qui distingue la domination violente de l'homme sur l'animal du respect pleinement humain — et donc aussi humanisant — envers les animaux, dont il importe de prendre en compte la dignité et la valeur. La voie du pathocentrisme trouve alors une certaine justification, mais sans occulter la perspective humaine seule à même de fonder une éthique et de la rendre socialement plausible.

\section{ABSTRACT}

The ethical and judicial standing of animals is the cause of many modern debates. The resolution of this question seems to lie behind the difficult choices between argumentative models that are apparently incompatible with each other. The author compares two major types of biocentricism: equalitarian biocentricism and hierarchal biocentricism, with two forms of anthropocentrism, radical or moderate. The results are that the anthropocentrical model has considerable advantages, that is, if there is a fundamental revision in the sense of an anthopo-rationality that distinguishes between the violent domination of man on 
animal life from the responsibility and respect of human beings toward animals, which need to be considered with dignity and with great value. The path to pathocentricism thus justified, should not prevent human perspective to create an ethic that is socially adequate.

(C) Revue Théologiques 2002. Tout droit réservé. 